\title{
Atitudes para 0 autocuidado em diabetes mellitus tipo 2 na Atenção Primária
}

\author{
Attitudes towards self-care in type 2 diabetes mellitus in primary care \\ Actitudes de autocuidado en diabetes mellitus tipo 2 en la Atención Primaria \\ Laura Barbosa Nunes ${ }^{1}$ ® https://orcid.org/10000-0002-6221-8406. \\ Jéssica Caroline dos Santos ${ }^{1}$ in https://orcid.org/0000-0001-8044-1276 \\ Illka Afonso Reis ${ }^{1}$ if https://orcid.org/0000-0001-7199-8590 \\ Heloisa de Carvalho Torres ${ }^{1}$ ib https://orcid.org/0000-0001-5174-3937
}

Como citar:

Nunes LB, Santos JC, Reis IA, Torres HC. Atitudes para 0 autocuidado em diabetes mellitus tipo 2 na Atenção Primária. Acta Paul Enferm. 2021;34:eAPE001765.

DOI

http://dx.doi.org/10.37689/actaape/2021A0001765

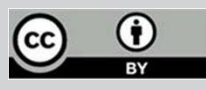

Descritores

Diabetes mellitus tipo 2; Atitude; Emoções; Autocuidado; Atenção primária à saúde

Keywords

Diabetes mellitus, type 2; Attitude; Emotions; Self care; Primary health care

Descriptores

Diabetes mellitus tipo 2; Actitud; Emociones; Autocuidado; Atención primaria de salud

Submetido 8 de Julho de 2020

Aceito

2 de Março de 2021

\section{Autora correspondente}

Heloísa de Carvalho Torres E-mail: heloisa@enf.ufmg.com

\section{Resumo}

Objetivo: Analisar as atitudes para 0 autocuidado de pessoas com diabetes tipo 2 na Atenção Primária.

Métodos: Estudo descritivo-exploratório, realizado com 18 pessoas com diabetes tipo 2 vinculadas a duas Unidades Básicas de Saúde, que participaram de entrevistas e grupos focais. Os conteúdos textuais foram processados no software IRaMuTeQ. Para avaliar a associação entre as variáveis categóricas, foi utilizado 0 teste qui-quadrado. Para todas as análises, foi considerado um nível de significância de 5\%.

Resultados: Os achados apresentaram uma classe semântica associada às atitudes para 0 autocuidado em diabetes mellitus tipo 2, e as outras quatro subclasses, com ideias complementares, decrescentes e lógicas, referiram-se aos aspectos emocionais, comportamentais, cognitivos e do autocuidado. Observou-se associação entre as subclasses e as variáveis sexo, idade e tempo diagnóstico.

Conclusão: Devem-se levar em consideração os aspectos emocionais e cognitivos, juntamente das variáveis sexo, idade e tempo diagnóstico, para a modificação das atitudes para o autocuidado em diabetes tipo 2.

\section{Abstract}

Objective: To analyze attitudes towards self-care of people with type 2 diabetes in Primary Care.

Methods: This is a descriptive-exploratory study, conducted with 18 people with type 2 diabetes linked to two Basic Health Units, who participated in interviews and focus groups. Text content was processed using the IRaMuTeQ software. To assess the association between categorical variables, the chi-square test was used. For all analyses, a significance level of $5 \%$ was considered.

Results: The findings presented a semantic class associated with attitudes towards self-care in type 2 diabetes mellitus, and the other four subclasses, with complementary, decreasing and logical ideas, referred to emotional, behavioral, cognitive and self-care aspects. An association was observed between the subclasses and the variables sex, age and time since diagnosis.

Conclusion: Emotional and cognitive aspects must be taken into account, together with the variables sex, age and time of diagnosis, for modifying attitudes towards self-care in type 2 diabetes.

\section{Resumen}

Objetivo: Analizar las actitudes de autocuidado de personas con diabetes tipo 2 en la Atención Primaria.

Métodos: Estudio descriptivo-exploratorio, realizado con 18 personas con diabetes tipo 2 vinculadas a dos Unidades Básicas de Salud, que participaron en entrevistas y grupos focales. Los contenidos textuales fueron procesados en el software IRaMuTeQ. Para evaluar la relación entre las variables categóricas se utilizó la prueba $\chi^{2}$ de Pearson. Se consideró un nivel de significación de $5 \%$ para todos los análisis. 
Resultados: Los resultados presentaron una clase semántica relacionada con las actitudes de autocuidado en diabetes mellitus tipo 2, y las otras cuatro subclases, con ideas complementarias, decrecientes y lógicas, estuvieron asociadas a los aspectos emocionales, de comportamiento, cognitivos y de autocuidado. Se observó una relación entre las subclases y las variables sexo, edad y tiempo de diagnóstico.

Conclusión: Deben tenerse en cuenta los aspectos emocionales y cognitivos, junto con las variables sexo, edad y tiempo de diagnóstico, para modificar las actitudes de autocuidado en diabetes tipo 2 .

\section{Introdução}

O diabetes mellitus tipo 2 é um dos principais problemas mundiais para o sistema de saúde pública, devido ao seu crescimento ascendente e ao elevado grau de morbimortalidade. ${ }^{(1)}$ Apresenta impacto sobre os aspectos psicológicos, quando em condição náo controlada, e pode trazer danos à saúde emocional das pessoas que lidam com a cronicidade no dia a dia, resultando na diminuição das práticas de autocuidado. $^{(2)}$

As atitudes para o autocuidado são reações emocionais que podem interferir na condição de saúde positiva ou negativamente. ${ }^{(3,4)}$ Por exemplo, a tristeza, a raiva e o medo levam a um estado de humor que interfere nas práticas de autocuidado, relacionadas à inatividade física, à perda de interesse pelo controle alimentar e à diminuição da adesão aos medicamentos. ${ }^{(5,6)}$

Estudo sobre a efetividade de um programa comportamental em diabetes mostrou que abordagens focadas na emoçáo e na cogniçáo afetaram as mudanças de comportamento, gerando benefícios psicológicos e físicos. ${ }^{(2)}$ Isso sugere que as atitudes vão além do aspecto emocional, podendo envolver aspectos cognitivo e comportamental, que devem ser considerados conjuntamente, para melhor convivência com a doença e manutenção do autocuidado. ${ }^{(4)}$

Compreender a multifatorialidade das atitudes é essencial para a promoção do autocuidado. ${ }^{(7,8)}$ As atitudes sáo compostas por determinantes afetivos (emoçóes), comportamentais e cognitivos (crenças),${ }^{(9)}$ podendo apresentar relaçáo com fatores sociodemográficos. ${ }^{(8)} \mathrm{O}$ ajustamento conjunto desses determinantes pressupóe o comportamento das pessoas com diabetes mellitus tipo 2 para o autocuidado, favorecendo o desenvolvimento de atitudes positivas.

Diante da magnitude do problema, conhecer as atitudes para o autocuidado das pessoas em relaçáo ao diabetes mellitus tipo 2 é essencial para reconhecer dificuldades e a melhor forma de ajustamento emocional. ${ }^{(6)}$ Os programas comportamentais em diabetes tipo 2 na Atenção Primária devem procurar valorizar náo somente as práticas de autocuidado, mas também as atitudes, visto que podem ser preditores para mudanças de comportamento. ${ }^{(2)}$ Ressaltase que as emoçóes devem ser trabalhadas em grupo e individualmente, respeitando o contexto de vida das pessoas com diabetes mellitus tipo $2 .^{(2)}$

Nesse sentido, identificar a relação dos fatores sociodemográficos com as atitudes para o autocuidado é essencial para que se possa promover programa comportamental em diabetes tipo 2 na Atenção Primária para orientação das práticas de autocuidado.

Assim, o objetivo deste estudo foi analisar as atitudes para o autocuidado de pessoas com diabetes tipo 2 na Atenção Primária.

\section{Métodos}

Trata-se de estudo descritivo-exploratório, realizado com 18 pessoas com diabetes mellitus tipo $2 \mathrm{de}$ ambos os sexos, com idade de 40 a 80 anos, vinculadas a duas Unidades Básicas de Saúde da regional leste do município de Belo Horizonte (MG), no período agosto a setembro de 2019. Os participantes foram incluídos no estudo por participarem do programa comportamental para orientação das práticas de autocuidado em diabetes e apresentarem interesse na educação para as práticas de autocuidado.

Os critérios de inclusão foram: ter idade de 40 a 80 anos (pois o diabetes mellitus tipo 2 acomete mais essa faixa etária) e apresentar complicaçôes crônicas (definidas como insuficiência renal, cegueira, amputação de membros, entre outras). ${ }^{(10)} \mathrm{Os}$ critérios de exclusão foram: ter incapacidade auditiva observada no primeiro encontro do programa comportamental e não conseguir contato telefônico, após três tentativas de ligaçóes para efetivar o convite para participar do grupo focal. 
Um roteiro foi elaborado para coletar informaçóes sociodemográficas (idade, sexo, renda e estado civil) de cada participante, bem como tempo de diagnóstico, por meio de entrevista face a face antes do grupo focal. Para resumir os dados coletados, foi utilizado o cálculo de frequência relativa, expressa em porcentagem.

Foram realizados dois grupos focais, ${ }^{(1)}$ sendo um em cada Unidade Básica de Saúde, coordenados por uma enfermeira e com apoio de uma nutricionista, que registrou em um diário de campo as comunicações não verbais, as posturas e as impressões dos participantes. Cada grupo focal foi constituído de forma homogênea, com participação de oito a dez pessoas com diabetes mellitus tipo 2 e duração de 1 hora.

Inicialmente, utilizou-se uma dinâmica de apresentação e aquecimento, na qual os participantes eram estimulados a refletir sobre si mesmos e a expressar suas emoçóes relacionadas ao autocuidado. Em seguida, foi aplicado o segundo passo do protocolo de mudança de comportamento, ${ }^{(12)}$ para nortear a discussão das atitudes para o autocuidado em diabetes, feito com as perguntas norteadoras: Como você se sente com essa situação de ter de cuidar da sua saúde (cuidar e controlar a doença)? Você sente [inserir os sentimentos expostos pela pessoa] por quê? A finalidade dessas duas questóes foi valorizar os aspectos emocionais para conduzir as pessoas à raiz do problema, além de auxiliar no desenvolvimento de estratégias que contribuem para adoção de atitudes positivas diante do autocuidado.

Os depoimentos dos participantes foram gravados e transcritos, e os conteúdos textuais foram processados no programa Interface de $\mathrm{R}$ pour les Analyses Multidimensionnelles de Textes et de Questionnaires (IRaMuTeQ). ${ }^{(13)}$

Antes do processamento pelo IRaMuTeQ, os depoimentos interpretados pelas autoras do estudo foram apreciados mediante a análise de conteúdo adaptada por Bardin, ${ }^{(14)}$ conforme a Classificação Hierárquica Descendente. Os conteúdos textuais foram codificados e processados pelo programa, no qual a distribuição de vocabulário pelas subclasses foi feita pela semelhança do vocabulário dos segmentos de texto. $\mathrm{Na}$ Classificação Hierárquica
Descendente, os segmentos de texto e o vocabulário foram correlacionados, formando um esquema hierárquico entre a classe e as subclasses. A origem das subclasses deve-se à retenção de, no mínimo, 70\% de segmentos de texto com palavras semelhante entre si e, ao mesmo tempo, com vocabulário diferente dos segmentos de outras subclasses. ${ }^{(13)}$

A relação entre a classe e as subclasses semânticas foi definida pela atribuição dos sentidos originados a partir da escuta e do compartilhamento de opinióes, valores, ideias e preferências entre os participantes referentes à modificação das atitudes para o autocuidado, sob intervenção dos aspectos emocionais e das variáveis sexo, idade e tempo de diagnóstico.

Após a Classificação Hierárquica Descendente simples, foram consideradas, para inserção em cada subclasse, as palavras cuja frequência se repetiu no conteúdo do texto. A formação das subclasses deu-se por meio dos segmentos de texto processados que apresentavam palavras homogêneas. Para a classificação e a relação das subclasses, os segmentos de texto foram agrupados quanto às ocorrências das palavras, o que resultou na criação de um dicionário com formas reduzidas. ${ }^{(13,15,16)}$

Utilizou-se o teste do qui-quadrado de Pearson para avaliar a associação entre as palavras e sua respectiva subclasse, bem como entre as variáveis categóricas dentro de cada subclasse (nível de significância de $5 \%) .{ }^{(16)}$

$\mathrm{O}$ estudo atendeu às normas de ética em pesquisa envolvendo seres humanos, conforme resolução 466/12. O projeto foi submetido ao Comitê de Ética em Pesquisa, com parecer 4.249.989.

\section{Resultados}

Das 18 (100\%) pessoas com diabetes mellitus tipo 2 entrevistadas, a maioria era do sexo feminino (61\%), casada (67\%), com renda familiar de um a dois salários mínimos ao mês (77\%). A média de idade foi de 66 anos (desvio-padrão de 10 anos). Com relação ao tempo diagnóstico, 61\% declararam ter diabetes há mais de 10 anos, enquanto $17 \%$ convivia com a doença há até 5 anos. 
Em relação aos depoimentos, os conteúdos textuais processados no programa apresentaram $98 \mathrm{seg}$ mentos de texto com potencial de análise de um total de 126, o que indica nível de aproveitamento de $77,6 \%$. A partir da leitura e da interpretação do corpus textual, por meio da análise das frequências das palavras, as autoras estabeleceram uma classe, referente às atitudes para o autocuidado em diabetes mellitus tipo 2, e quatro subclasses, com ideias complementares, lógicas e decrescentes: (4) Aspecto emocional; (3) Comportamental; (2) Cognitivo e (1) Autocuidado.

Os dados foram organizados em um dendograma, que ilustrou as relaçóes de proximidade e distanciamento entre as subclasses. Hierarquicamente, havia dois grandes níveis de partição. $\mathrm{O}$ primeiro distinguiu as subclasses 1, 2 e 3, de um lado, enquanto o segundo separou a subclasse 4 das demais. Assim, eram esperados que fossem mais semelhantes entre si, em termos de vocabulário, os conteúdos textuais pertencentes às subclasses 1,2 e 3 . Além disso, por ser a subclasse mais numerosa, envolvendo $35,5 \%$ do corpus textual, era possível intuir que a subclasse 3 fosse a mais heterogênea do conjunto.

Foi identificada associação significativa entre a ocorrência das palavras e as variáveis sexo, idade e tempo diagnóstico apenas dentro das subclasses (Figura 1).
A adoção de atitudes positivas ou negativas, relacionadas à emoção, foi observada no primeiro nível da partição na subclasse 4, que, por sua vez, permeou o segundo nível de partição, referente às subclasses 3, 2 e 1, ou seja, no aspecto comportamental e, em menor porcentagem, no aspecto cognitivo e no autocuidado da pessoa com diabetes mellitus tipo 2. Essa conexão entre as subclasses sugeriu que a construção multifatorial das atitudes envolveu os quatro aspectos, sendo que a emoção podia comprometer a prontidão para o autocuidado.

\section{Aspectos emocionais}

Os conteúdos textuais dessa subclasse retrataram as emoçôes positivas e negativas que foram apresentadas pelas pessoas com diabetes do sexo feminino. Apesar de não ter sido a subclasse significativa do conjunto (30,3\% de segmentos de texto), foi a que se destacou quanto aos vocábulos e aos conteúdos dos segmentos de texto representados pela interferência sobre as demais subclasses.

Com base nas palavras e nas ideias encontradas nos segmentos do texto, o aspecto emocional favoreceu a reflexão sobre as atitudes de autocuidado relacionadas à tristeza decorrente dos maus hábitos alimentares, como o consumo de massas, doces, gorduras e bebidas açucaradas. A tristeza também

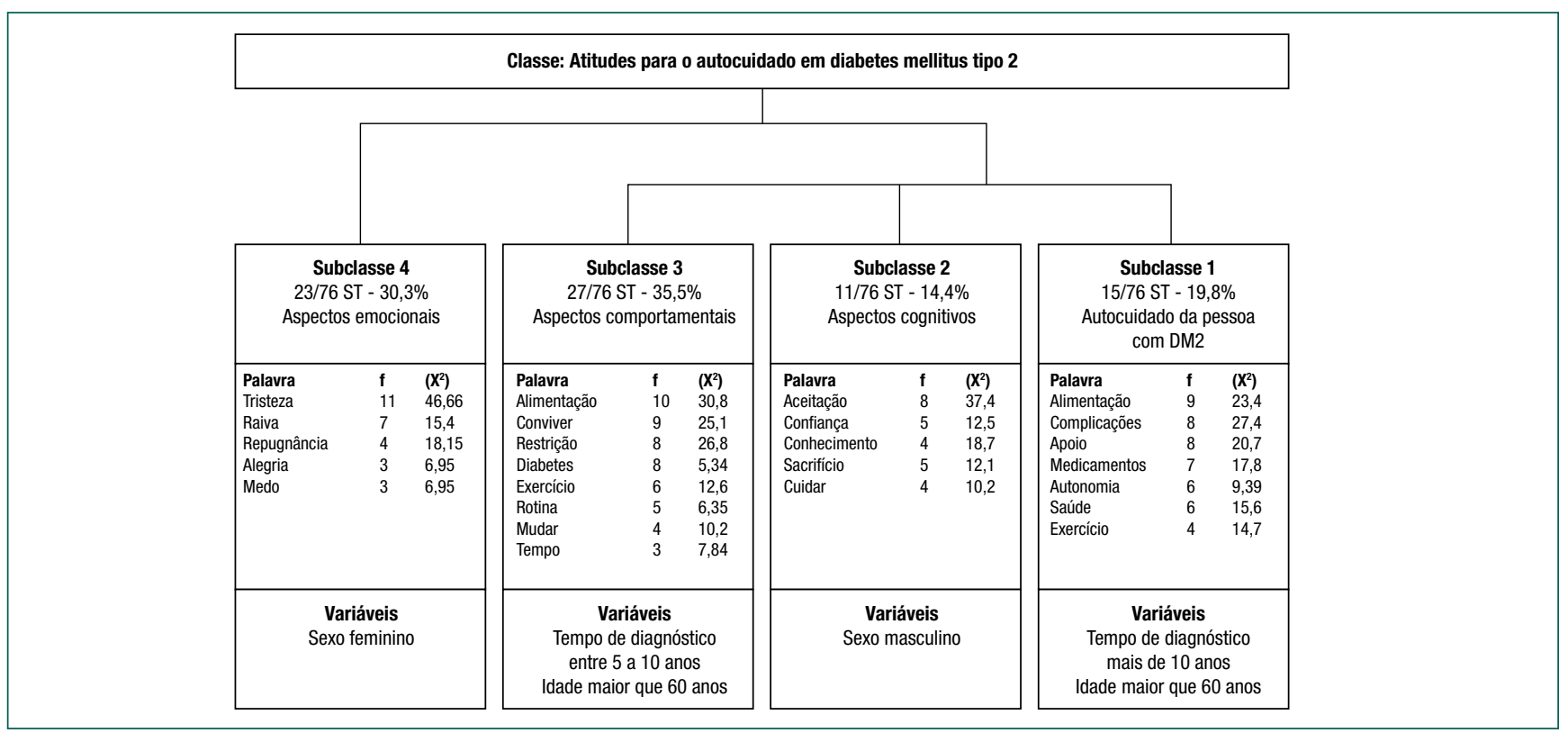


esteve vinculada à compreensão de que o esforço no seguimento alimentar restritivo e na prática de atividade física não representou cura do diabetes mellitus tipo 2 - apenas controle.

A raiva foi apontada pelas pessoas como decorrente da sensação de que a doença controlava sua vida, impondo restriçóes a alimentos prazerosos, a exemplo dos doces consumidos em ocasióes festivas. Também esteve relacionada ao uso de medicamentos várias vezes ao dia.

Outras reaçóes emocionais apontadas foram a repugnância, pela resistência à mudança de comportamento, e o medo das complicaçóes, como pé diabético, retinopatia, neuropatia e nefropatias. $\mathrm{O}$ medo também estava vinculado à capacidade de realizaçáo do autocuidado relacionado à alimentação, à atividade física e ao uso correto de medicamentos. Evidenciou-se, ainda, a alegria pelo despertar de atitudes positivas em relação ao autocuidado.

\section{Aspectos comportamentais}

Essa subclasse envolveu 35,5\% de segmentos de texto, e seu conteúdo foi expressivo entre participantes com tempo diagnóstico entre 5 e 10 anos e com idade acima de 60 anos. As palavras significativas evidenciaram a necessidade da mudança de comportamento para que se alcance a prontidão para o autocuidado, associado à alimentação saudável e à prática de atividade física. A restrição alimentar foi considerada a principal dificuldade encontrada, seguida da prática de atividade física.

Verificou-se que os comportamentos adotados para manejo do diabetes mellitus tipo 2, somados aos aspectos cognitivos, foram motivados pelos aspectos emocionais verificados no primeiro nível da partição, especialmente a tristeza e a raiva, interferindo diretamente nas práticas de autocuidado, o que é representado pela ligação indireta entre as subclasses 4 e 3 e direta entre as subclasses 3, 2 e 1 .

Percebeu-se que a mudança de comportamento é um processo lento que acontece à medida que se percebe a melhora da saúde, o que, acompanhado de emoçóes positivas, favorece o aumento da confiança verificada no aspecto cognitivo.

\section{Aspectos cognitivos}

Essa subclasse constituiu-se de 14,4\% dos segmentos de texto vinculados ao sexo masculino. Diz respeito às crenças, às informaçóes, às ideias e ao conhecimento que as pessoas relataram referentes à condição crônica e ao seu manejo. Observou-se que a aceitação da doença e a confiança na realização das práticas de autocuidado remeteram ao bem-estar e à possibilidade de novos caminhos, contribuindo para a motivação e a responsabilidade de cuidar da própria saúde, o que foi evidenciado pela ligação direta entre essa subclasse e a subclasse do autocuidado.

\section{Autocuidado da pessoa com diabetes mellitus tipo 2}

A subclasse do autocuidado, constituída por $19,8 \%$ dos segmentos, foi composta pelos depoimentos de participantes com tempo diagnóstico superior a 10 anos e idade acima de 60 anos. Esteve indiretamente ligada à subclasse do aspecto emocional e diretamente às subclasses dos aspectos comportamental e cognitivo, mostrando que as atitudes que a pessoa adotava envolviam o aspecto emocional, comportamental e cognitivo, repercutindo no autocuidado.

As sentenças relacionadas a essa subclasse evidenciaram que o autocuidado referente à alimentação saudável proporcionou novas práticas de autocuidado, como o uso correto dos medicamentos, contribuindo para uma atitude positiva no manejo do diabetes. $\mathrm{O}$ apoio familiar diminuiu o medo e a insegurança em relação às complicaçóes e foi representativo para a aceitação da condição crônica, conforme apontado na subclasse do aspecto cognitivo.

\section{Discussão}

As características sociodemográficas dos participantes desta pesquisa assemelharam-se às de outros estudos realizados com pessoas com diabetes mellitus tipo 2, a exemplo da predominância do sexo feminino, ${ }^{(8,17)}$ com companheiro, ${ }^{(18)}$ idade superior a 50 anos, ${ }^{(9,16)}$ baixa renda e tempo diagnóstico acima de 10 anos. $^{(7,19)}$

Neste estudo, a análise das atitudes para o autocuidado de pessoas com diabetes mellitus tipo 2 evi- 
denciou as quatro subclasses que as determinaram: aspectos emocional, comportamental, cognitivo e de autocuidado da pessoa com diabetes mellitus tipo 2. Compreender cada um desses aspectos e os fatores sociodemográficos relacionados é essencial para o entendimento das atitudes nos diversos contextos, superando as adversidades impostas pela condição, em busca da adesão ao autocuidado. ${ }^{(7,8)}$

Corroborando os achados desta pesquisa, estudo sobre o conhecimento das atitudes e sua influência na adesão a estratégias para autocuidado de pessoas com diabetes mellitus tipo 2 revelou as atitudes quanto aos componentes cognitivo (conhecimento e crença), comportamental (comportamento e autocuidado) e afetivo (estado emocional). ${ }^{(4)}$

Sobre as subclasses, os aspectos emocionais interferiram nos aspectos comportamentais, cognitivos e de autocuidado, por meio de diferentes emoçóes, principalmente tristeza e raiva. As emoçóes surgiram diante da necessidade de cuidados específicos, tornando-se evidentes nas pessoas do sexo feminino que não estavam preparadas para conviver com as limitaçóes associadas ao diabetes mellitus tipo 2.

Esse achado assemelhou-se ao de estudos anteriores. Houve, contudo, predominância de desequilíbrio emocional no sexo feminino. Percebe-se que os efeitos da inatividade laboral decorrente da idade tendem a potencializar o sentimento de improdutividade, somando-se à desesperança e ao medo diante da capacidade de realizar práticas de autocuidado. Acrescem-se a isso as diferenças biológicas entre os sexos, no que tange às reaçóes emocionais. ${ }^{(6,8,20)}$

A tristeza, evidente na subclasse dos aspectos emocionais, associou-se às restriçóes no controle alimentar, devido a hábitos prejudiciais e à perda do "eu" anterior. Entende-se que identificar antigos hábitos considerados destrutivos e reconhecer a diferença entre como se deve viver e como se vive parecem ser processos de difícil superação. ${ }^{(5,21)}$

A literatura aponta que a tristeza deve-se ao desânimo para realizar o controle, o tratamento e a prevenção das complicaçóes e à compreensão de que o esforço não representa uma cura em si. ${ }^{(22)}$ Contudo, observou-se que a tristeza tende a desaparecer à medida que surge a aceitação do diabetes, evidenciada no aspecto cognitivo.
A raiva, outro componente emocional, mostrou que o cerceamento da liberdade para se alimentar como e quando desejar e o uso de medicamentos várias vezes ao dia trouxeram insegurança e perspectiva desoladora para o convívio com o diabetes, que parece subtrair o prazer de viver. ${ }^{(20,23)}$ As restriçóes impostas tiram a liberdade de fazer o que se tem vontade. ${ }^{(24)}$

$\mathrm{Na}$ subclasse do aspecto comportamental, o tempo diagnóstico entre 5 e 10 anos e a idade acima de 60 anos favoreceram a mudança de comportamento, associados ao seguimento alimentar saudável e à prática de atividade física, com consequentes atitudes positivas para autocuidado, visto que as pessoas demonstraram seriedade na convivência com o diabetes. ${ }^{(25)}$ Contudo, observou-se resistência para a construção desses comportamentos, o que pode estar relacionado às dificuldades emocionais nos processos iniciais para aceitação do diabetes. ${ }^{(22)}$

Em consonância com esses resultados, estudo mostra que o tempo diagnóstico pode ter impacto significativo nas emoçóes das pessoas com diabetes mellitus tipo 2, considerando que as atitudes podem ser modificadas ao longo dos anos, tornando-se positivas em cada fase da vida. ${ }^{(26)}$ Entretanto, o tempo de diabetes e a idade avançada são fatores para o aparecimento de complicaçôes, levando à pouca motivação para o autocuidado e à dependência na realização das atividades, o que pode contribuir para uma atitude negativa referente ao comportamento. ${ }^{(8)}$

Quanto ao aspecto cognitivo, observou-se que a aceitação do diabetes constituiu um processo de adaptação que favoreceu o enfrentamento das limitações, especialmente no seguimento de um plano alimentar saudável e com evocação de emoçôes, como a alegria. ${ }^{(27)}$

Destaca-se que o modo como cada pessoa convive com o diabetes mellitus tipo 2 depende das experiências individuais, tornando-se, muitas vezes, necessária a desconstrução de crenças, valores e costumes relacionados aos aspectos da vida cotidiana, até então aceitos, para se alcançar um ajustamento emocional, social e fisiológico durante o tratamento. ${ }^{(28)}$

Verificou-se também o aumento da confiança à medida que os esforços se concretizavam, acompanhada da sensaçáo de retomada do controle da vida e do retorno da liberdade para fazer escolhas seguras 
e confortáveis. ${ }^{(3,21)}$ As emoçóes positivas são decorrentes da percepção da melhora da saúde e da aquisição de habilidades de autocuidado, fazendo com que a pessoa com diabetes mellitus tipo 2 enxergue novas possibilidades diante da vida. ${ }^{(5,29)}$

Ser homem foi fator de proteção à atitude negativa para o autocuidado, verificada no aspecto cognitivo. Isso sugere que as pessoas do sexo masculino souberam lidar melhor com a desconstrução das crenças e estavam mais confiantes no tratamento. Esse resultado corrobora estudo recente sobre atitudes e fatores sociodemográficos relacionados ao autocuidado, que apontou que ser homem foi significativamente associado à adaptação psicológica, fazendo-os possuir atitudes positivas diante do diabetes mellitus tipo 2. ${ }^{(18)}$

Em relação à subclasse do autocuidado, o tempo de diagnóstico superior a 10 anos e a idade acima de 60 anos foram fatores facilitadores para o desenvolvimento do autocuidado, uma vez que pessoas com essas características demonstraram estar mais conectadas com suas necessidades e suas metas. ${ }^{(18)}$ Isso aponta que as pessoas têm capacidade cognitiva preservada, apesar da idade. Entretanto, a literatura evidencia que a idade é considerada fator impeditivo para aquisição de competências de autocuidado, visto que, com o avançar da idade, ocorrem declínios cognitivo e motor. ${ }^{(18)}$

$\mathrm{O}$ apoio familiar, observado na subclasse de autocuidado, mostrou-se importante para aceitação do diabetes, reconhecimento das restriçóes alimentares e construção conjunta das práticas de autocuidado. (30) Coerentemente com esses resultados, autores relataram que pessoas com diabetes mellitus tipo 2, que possuíam apoio, aderiram à alimentação saudável 1,27 vez a mais que aquelas que náo possuíam. ${ }^{(31)}$

Com base neste estudo, entende-se que as atitudes interferem na propensão de a pessoa com diabetes mellitus tipo 2 adotar determinados comportamentos para o autocuidado. ${ }^{(3)}$ Contudo, ignorar o contexto emocional da atitude implica não garantir assistência adequada às necessidades de cada um. A atitude positiva para o autocuidado pode ser uma realidade ilusória enquanto não houver ajustamento conjunto dos aspectos emocional, comportamental, cognitivo e de autocuidado..$^{(2-4)}$

Considera-se como limitação do estudo as dificuldades de comparecimento dos usuários aos encontros do grupo focal, que, em sua maioria, estiveram relacionadas com os afazeres domésticos das donas de casa e consultas agendadas no horário do encontro. Podemse citar também dificuldades relacionadas aos participantes, como o não aferimento do real grau cognitivo e o estado emocional da pessoa abalado por problemas pessoais, o que significa que os achados refletem a situação de parcela limitada da população, restringindo a possibilidade de generalizaçáo dos resultados.

\section{Conclusão}

Este estudo mostra a importância de levar em consideração os aspectos emocionais e cognitivos, juntamente das variáveis sexo, idade e tempo diagnóstico, para a modificação das atitudes para o autocuidado em diabetes tipo 2 .

\section{Agradecimentos}

À Coordenação de Aperfeiçoamento de Pessoal de Nível Superior (CAPES) pela concessão da bolsa de Doutorado a Laura Barbosa Nunes. Ao Conselho Nacional de Desenvolvimento Científico e Tecnológico (CNPq) - processos 303250/20194 e 432824/2016-2, e à Fundação de Amparo à Pesquisa de Minas Gerais (FAPEMIG) - processo APQ-03865-16, pelo financiamento deste estudo.

\section{Colaborações}

Nunes LB, Santos JC, Reis IA e Torres HC declaram que contribuíram com a concepção do projeto, a análise e a interpretação dos dados, a revisão crítica relevante do conteúdo intelectual e a aprovação da versão final a ser publicada.

\section{Referências}

1. Sociedade Brasileira de Diabetes (SBD). Diretrizes da Sociedade Brasileira de Diabetes 2019-2020. São Paulo: Clannad; 2018 [citado 2020 Dez 10]. Disponível em: https://www.diabetes.org.br/ profissionais/images/DIRETRIZES-COMPLETA-2019-2020.pdf 
2. Chew BH, Vos RC, Shariff Ghazali S, Shamsuddin NH, Fernandez A, Mukhtar $F$, et al. The effectiveness of a value-based EMOtion-cognitionFocused educatlonal programme to reduce diabetes-related distress in Malay adults with Type 2 diabetes (VEMOFIT): study protocol for a cluster randomised controlled trial. BMC Endocr Disord. 2017;17(1):22.

3. Abolghasemi $\mathrm{R}$, Sedaghat M. The patient's attitude toward type 2 diabetes mellitus, a qualitative study. J Relig Health. 2015;54(4):1191-205.

4. Pupko VB, Azzollini S. Actitudes, afrontamiento y autocuidado en pacientes con diabetes tipo 2 / Attitudes, ways of coping and selfcare in patients with type 2 diabetes. Rev Argent Salud Publica. 2012;3(10):15-23.

5. Gabre M, Sundström BW, Olausson S. A little good with the bad: newly diagnosed type 2 diabetes patients' perspectives on self-care: a phenomenological approach. Nord J Nurs Res. 2019;39(1):20-8.

6. Wong EM, Afshar R, Qian H, Zhang M, Elliott TG, Tang TS. Diabetes distress, depression and glycemic control in a Canadian-Based Specialty Care Setting. Can J Diabetes. 2017;41(4):362-5.

7. Assunção SC, Fonseca AP, Silveira MF, Caldeira AP, Pinho L. Knowledge and attitude of patients with diabetes mellitus in Primary Health Care. Esc Anna Nery. 2017;21(4):e20170208.

8. Borba AK, Arruda IK, Marques AP, Leal MC, Diniz AD. Knowledge and attitude about diabetes self-care of older adults in primary health care. Cien Saude Colet. 2019;24(1):125-36.

9. Ajzen I. The theory of planned behavior. Organ Behav Hum Decis Process. 1991;50(2):179-211.

10. International Diabetes Federation (IDF). IDF Diabetes Atlas. 8th ed. Belgium: IDF; 2017 [cited 2020 Dec 10]. Available from: http:// fmdiabetes.org/wp-content/uploads/2018/03/IDF-2017.pdf

11. Gomes S, Barbosa EF; Educativa: Instituto de Pesquisas e Inovações Educacionais. Uma técnica de grupos focais para obtenção de dados qualitativos. Belo Horizonte: Instituto de Pesquisa e Inovações Educacionais; 1999 [citado 2020 Dez 10]. Disponível em: http://www. tecnologiadeprojetos.com.br/banco_objetos/\%7B9FEA090E-98E949D2-A638-6D3922787D19\%7D_Tecnica\%20de\%20Grupos\%20 Focais\%20pdf.pdf

12. Chaves FA, Cecilio SG, Reis IA, Pagano AS, Torres HC. Translation and cross-cultural adaptation of the behavior change protocol for educational practices in diabetes mellitus. Rev Lat Am Enfermagem. 2019;27:e3164.

13. Camargo BV, Justo AM. IRAMUTEQ: um software gratuito para análisede dados textuais. Temas Psicol. 2013;21(2):513-8.

14. Bardin L. Análise de conteúdo. Lisboa: Edições 70; 2011.

15. Berri $B$, Castro $A$, Camargo BV. Representações sociais relacionadas às práticas de rejuvenescimento. Psicol Pes UFJF. 2016;10(2):21-30.

16. Lahlou S. Text mining methods: an answer to Chartier and Meunier. Papers Soc Represent. 2012;20:38:1-7 [cited 2020 Dec 10]. Available from: http://eprints.lse.ac.uk/46728/1/Text\%20mining\%20 methods(Isero).pdf

17. Karimy M, Koohestani HR, Araban M. The association between attitude, self-efficacy, and social support and adherence to diabetes self-care behavior. Diabetol Metab Syndr. 2018;10:86.
18. Niguse H, Belay G, Fisseha G, Desale T, Gebremedhn G. Self-care related knowledge, attitude, practice and associated factors among patients with diabetes in Ayder Comprehensive Specialized Hospital, North Ethiopia. BMC Res Notes. 2019;12(1):34.

19. Cortez DN, Reis IA, Souza DA, Macedo MM, Torres HC. Complications and the time of diagnosis of diabetes mellitus in primary care. Acta Paul Enferm. 2015;28(3):250-5.

20. Nanayakkara N, Pease A, Ranasinha S, Wischer N, Andrikopoulos S, Speight J, et al. Depression and diabetes distress in adults with type 2 diabetes: results from the Australian National Diabetes Audit (ANDA) 2016. Sci Rep. 2018;8(1):7846.

21. Ledesma-Delgado ME, Oros-Elizarraras E, Ávila-Sansores GM, Ruvualcaba-Palacios G. Respuestas emocionales de las mujeres que viven con diabetes mellitus tipo 2. Rev Enferm Inst Mex Seguro Soc. 2014;22(2):85-92.

22. Hernández-Jiménez S, García-Ulloa AC, Bello-Chavolla OY, AguilarSalinas CA, Kershenobich-Stalnikowitz D; Group of Study CAIPaDi. Long-term effectiveness of a type 2 diabetes comprehensive care program. The CAIPaDi model. Diabetes Res Clin Pract. 2019;151:12837.

23. Péres DS, Franco LJ, Santos MA. Comportamento alimentar em mulheres portadoras de diabetes tipo 2. Rev Saúde Pública. 2006;40(2):310-7.

24. Schinckus L, Dangoisse F, Van den Broucke S, Mikolajczak M. When knowing is not enough: Emotional distress and depression reduce the positive effects of health literacy on diabetes self-management. Patient Educ Couns. 2018;101(2):324-30.

25. Cortez DN, Santos JC, Macedo MM, Souza DA, Reis IA, Torres HC. Efeito de um programa educacional em empoderamento do autocuidado para cumprimento de metas em diabetes. Ciencia $Y$ Enfermeria. 2018;24(3):23-32.

26. Ausili D, Rossi E, Rebora P, Luciani M, Tonoli L, Ballerini E, et al. Socio-demographic and clinical determinants of self-care in adults with type 2 diabetes: a multicentre observational study. Acta Diabetol. 2018;55(7):691-702.

27. Wu FL, Tai HC, Sun JC. Self-management Experience of Middle-aged and Older Adults With Type 2 Diabetes: A Qualitative Study. Asian Nurs Res (Korean Soc Nurs Sci). 2019;13(3):209-15.

28. Bertolin DC, Pace AE, Cesarino CB, Ribeiro RC, Ribeiro RM. Psychological adaptation to and acceptance of type 2 diabetes mellitus. Acta Paul Enferm. 2015;28(5):440-6.

29. Sarfati D, McLeod M, Stanley J, Signal V, Stairmand J, Krebs J, et al. BetaMe: impact of a comprehensive digital health programme on $\mathrm{HbA} 1 \mathrm{C}$ and weight at 12 months for people with diabetes and pre-diabetes: study protocol for a randomised controlled trial. Trials. 2018;19(1):161.

30. Cruz DS, Silva KL, Souza JT, Nóbrega MM, Reichert AP, Marques DK, et al. Experiences of adolescents with diabetes mellitus from the perspective of the ethics of alterity. Acta Paul Enferm. 2018;31(2):1306.

31. DiMatteo MR. Social support and patient adherence to medical treatment: a meta-analysis. Health Psychol. 2004;23(2):207-18. 